\title{
Interacting with the fifth dimension
}

\begin{abstract}
Some new five dimensional minimal scalar-Einstein exact solutions are presented. These new solutions are tested against various criteria used to measure interaction with the fifth dimension. The first type of five dimensional solutions has the fifth dimension entering the scalar field in a similar manner to the null coordinates. The second has $d=4$ spacetime the same as in the $d=4$ solution but has cross terms between the fifth dimension and the null coordinates. The third has a conformal factor multiplying the $d=4$ solution.
\end{abstract}

Keywords: dimension; scalar field; spacetime; horizons; einstein solutions; vector field
Volume 2 Issue I - 2018

\section{Roberts MD}

Theoretical physicist, Wonersh Park, UK

Correspondence: Mark D Roberts, Theoretical physicist, 54 Grantley Avenue, Wonersh Park, GU5 0QN, UK, Email robemark@gmail.com

Received: January 25, 2018 | Published: February 15, 2018

\section{Introduction}

The use of the scalar-Einstein field equations and their solutions seems to have gone through four, perhaps related, stages. In the first stage $^{1}$ solutions were sought which it was hoped would represent an elementary particle such as a meson. How the scalar field decays from such solutions might effect the Yukawa potential has been discussed. by Roberts ${ }^{2}$ In the second stage it was noticed that most scalar-Einstein solutions do not have event horizons. It was shown that no event horizons happen under fairly general conditions in the static case. ${ }^{3}$ In the non-static case there are imploding solutions which create curvature singularities out of nothing, ${ }^{4,5}$ these examples have no overall mass, but there is a mass in the corresponding conformalscalar solutions. ${ }^{6}$ In the third stage exact scalar-Einstein solutions where found to be critical cases in the numerical study of stellar collapse. $^{7}$ In the fourth stage exact scalar-Einstein solutions were canonically quantized. ${ }^{8}$

Contemporary attempts at quan- tum gravity and unification usually involve more than the observable four dimensions. ${ }^{9,10}$ It is possible that models in five dimensions might provide testable cosmological models Randall L \& Sundrum $\mathrm{R}^{11,12}$ or be testable on the scale of the solar system by Wesson, Mashhoon \& Lui ${ }^{13}$ or perhaps be testable microscopically. ${ }^{14}$ To build a five dimensional model in addition to an exact solution one needs to prescribe another piece of information: how the four dimensional spacetime is embedded in the five dimensional spaces. This is typically done by requiring that the four dimensional spacetime is a four dimensional surface in a five dimensional space, this can be achieved by choosing a normal vector field $n^{a}=\delta_{\chi}^{a}$ to the surface. Once five dimensional scalar-Einstein solutions have been found there turns out to be many in equivalent ways of doing this and it is not immediate which the best is. For simplicity here mainly solutions to the field equations $R_{a b}=2 \varphi_{a} \varphi_{b}$ are discussed. In particular these field equations and spherical symmetry require $R_{\theta \theta}=0$ so that there is no self-interaction, such as mass, for the scalar field, and also there is no cosmological constant present, both of these require $R_{\theta \theta} \alpha g_{\theta \theta}$. The cosmological con- stant is often taken to be related to a brane tension, so that the examples here are for zero tension.

Most calculations were done using Maple 9.

\section{The four dimensional solutions}

In single null coordinates the line element is

$$
d s_{4}^{2}=-(1+2 \sigma) d v^{2}+2 d v d r+r(r-2 \sigma v) d \Sigma_{2}^{2}, d \Sigma_{2}^{2}=d \theta^{2}+\sin (\theta)^{2} d \varphi^{2} .
$$

The scalar field takes the form

$$
\varphi=\frac{1}{2} \ln \left(1-\frac{2 \sigma v}{r}\right) .
$$

To transformation to double null coordinates use

$$
u \equiv(1+2 \sigma) v-2 r
$$

So that the line element becomes

$$
d s_{4}^{2}=-d u d v+Y^{2} d \Sigma_{2}^{2}, Y^{2} \equiv \frac{1}{4}((1+2 \sigma) v-u)((1-2 \sigma) v-u)
$$

Where $Y$ is the luminosity distance. In double null coordinates the scalar field takes the form

$$
\varphi=\frac{1}{2} \ln \left(\frac{(1-2 \sigma) v-u}{(1+2 \sigma) v-u}\right)
$$

Roughly speaking a Killing vector is a vector which points in a direction that space does not change, so if you go in its direction everything looks the same. Examples are time like killing vectors which is part of the requirement as to whether a spacetime is static and various rotational killing vectors. Homethetic means that where there was a zero for a Killing vector there is now a term proportional to the metric, so that going in the direction of the Killing vector things change by a constant amount. A gradient vector is a vector which is just the derivative of a single scalar, in general several scalars are needed. The solution has a homothetic gradient Killing vector with conformal factor $-2 c$ and potential, norm, acceleration, expansion, vorticity and orthogonality

$$
V=\operatorname{cuv}, V^{a} V_{a}=2 c V, A^{a}=c V^{a}, \Theta=-8 c, \omega=0, V^{a} \varphi_{a}=0 .
$$

The Ricci tensor is a two index contraction of the four index Riemann tensor, it can be further contracted to give the Ricci scalar. The Riemann tensor is constructed from the first and second derivatives of the metric and is a measure of the curvature. The general relativity field equations which related matter to geometry can be expressed in terms of either the Ricci tensor or Einstein tensor. Here the Ricci scalar is simply expressed 


$$
R=\frac{2 \sigma^{2} u v}{Y^{4}}
$$

The Weyl tensor is constructed from the Riemann tensor. In general relativity its contractions vanish and it is conformally invariant, which means that if the metric is multiplied by a function it remains the same. Using the preferred vector field $E[v]_{v v}=\frac{1}{3} R$, the magnetic part of the Weyl tensor vanishes, and the electric part has one component

$$
E[v]_{v v}=\frac{1}{3} R
$$

With $X_{v} E[v]_{a b}=0$. Using the preferred vector field $V$ the square root of the completely transected Bell-Robinson tensor is a measure of the gravitational energy and is

$$
\sqrt{B[V]}=\frac{\sqrt{22^{4}}}{3} c^{2} \sigma u v R
$$

Substituting into the definition of the Weyl tensor the product invariants obey

$$
\text { Weyl }^{2}=\text { Rie }^{2}-\frac{4}{d-2} \text { Ricci }^{2}+\frac{2}{(d-1)(d-2)} R^{2} .
$$

The product invariants can be expressed in terms of the Ricci scalar

$$
R_{1}=3.2^{-4} R^{2}, R_{2}=-3.2^{-6} R^{3}, R_{3}=3.7 .2^{-10} R^{4},
$$

As can the non-vanishing Carminati-McLenaghan ${ }^{15}$ invariants

$$
\begin{gathered}
R_{1}=3.2^{-4} R^{2}, R_{2}=-3 \cdot 2^{-6} R^{3}, R_{3}=3 \cdot 7 \cdot 2^{-10} R^{4}, \\
\Re\left(M_{2}\right)=M_{3}=3^{-1} \cdot 2^{-5} R^{4}, M_{4}=3^{-1} \cdot 2^{-8} R^{5}, \Re\left(M_{5}\right)=3^{-2} \cdot 2^{-6} R^{5} . \\
\Re\left(M_{2}\right)=M_{3}=3^{-1} \cdot 2^{-5} R^{4}, M_{4}=3^{-1} \cdot 2^{-8} R^{5}, \mathfrak{R}\left(M_{5}\right)=3^{-2} \cdot 2^{-6} R^{5} .
\end{gathered}
$$

\section{The first five dimensional solutions}

In double null coordinates the first five dimensional generalization of the four dimensional solution is

$$
\begin{gathered}
d s_{5}^{2}=-\frac{1}{1-\alpha \beta} d u d v+Y^{2} d \Sigma_{2}^{2}+d \chi^{2} \\
4 Y^{2} \equiv((1+2 \sigma) v-u+2 \alpha \chi)((1-2 \sigma) v-u+2 \beta \chi), \\
\varphi=\frac{1}{2} \ln \left(\frac{(1-2 \sigma) v-u+2 \beta \chi}{(1+2 \sigma) v-u+2 \alpha \chi}\right),
\end{gathered}
$$

The solution has a homothetic gradient Killing vector with conformal factor $c$ and potential, norm, acceleration and expansion

$$
V=\frac{c}{2 \alpha \beta-1} u v+\frac{c}{2} \chi^{2}, \operatorname{norm}[V]=2 c V, A^{a}=c V^{a}, \Theta=5 c
$$

And the vorticity vanishes. Defining

$$
\gamma \equiv(1-2 \sigma) \alpha-(1+2 \sigma) \beta
$$

The Ricci scalar is given by

$R=\left[16 \sigma^{2}(1-\alpha \beta) u v+((\alpha-\beta) u-\gamma v)^{2}+4(1-\alpha \beta)(2 \sigma((\alpha-\beta) u+\gamma v)+(\alpha-\beta) \gamma \chi) \chi\right] /\left(8 Y^{4}\right)(16)$

The product invariants are again completely determined by the Ricci scalar, however contrasting with (11) this time

$$
\text { Rie }^{2}=3 R^{2}, \text { Ricci }^{2}=R^{2}, \text { Weyl }^{2}=\frac{11}{6} R^{2},
$$

So that the fraction multiplying the Weyl invariant has increased by $1 / 2$ from $4 / 3$ to $11 / 6$, signifying that there is more gravitational field present.

The relationship between the product invariants (10) is obeyed by both (11) and (17).

Projecting onto a surface

$$
n_{a}=\delta_{a}^{\chi},
$$

Which has vanishing acceleration and rotation, and expansion, shear and extrinsic curvature given by

$$
\begin{aligned}
& \Theta=-\frac{1}{2 Y^{2}}(\alpha((1-2 \sigma) v-u)+4 \alpha \beta \chi+\beta((1+2 \sigma) v-u \\
& \sigma_{a b}=-\frac{1}{6}\left(-2 \delta_{a b}^{(u v)} g_{u v}+\delta_{a b}^{\theta \theta} g_{\theta \theta}+\delta_{a b}^{\varphi \varphi} g_{\varphi \varphi}\right) \Theta, \sigma(n)=-\frac{\sqrt{10}}{24 Y^{2}} \Theta \\
& K_{a b} \equiv g_{a}^{c} n_{b ; c}=-\frac{1}{2}\left(\delta_{a b}^{\theta \theta} g_{\theta \theta}+\delta_{a b}^{\varphi \varphi} g_{\varphi \varphi}\right) \Theta
\end{aligned}
$$

Respectively. The projected Weyl tensor is

$$
E_{e f} \equiv C_{a c b d} n^{c} n^{d} g_{e}^{a} g_{f}^{b},
$$

Here

$$
E_{u u}=-\frac{1}{3} R_{u u}, E_{v v}=-\frac{1}{3} R_{v v}, E_{u v}=+\frac{1}{3} R_{u v}-\frac{1}{4} R g_{u v}, E_{\theta \theta}=g_{\theta \theta}\left(\frac{1}{4} R+\frac{2}{3}(1-\alpha \beta) R_{u v}\right)
$$

Transferring to single null coordinates using (3), further changing coordinates using

$$
r^{\prime}=r+\alpha \chi, v^{\prime}=v+\frac{(\alpha-\beta) \chi}{2 \sigma}, \sigma \neq 0,
$$

And dropping the primes, the solution becomes

$$
\begin{aligned}
& d s^{2}=-\frac{(1+2 \sigma)}{(1-\alpha \beta)} d v^{2}+\frac{2}{(1-\alpha \beta)} d v d r+r(r-2 \sigma v) d \Sigma_{2}^{2} \\
& +\frac{(\alpha-(1+2 \sigma) \beta)}{\sigma(1-\alpha \beta)} d v d \chi-\frac{(\alpha-\beta)}{\sigma(1-\alpha \beta)} d r d \chi+\left(1-\frac{(\alpha-\beta) \gamma}{4 \sigma^{2}(1-\alpha \beta)}\right) d \chi^{2}, \\
& \varphi=\frac{1}{2} \ln \left(1-\frac{2 \sigma v}{r}\right)
\end{aligned}
$$

With $\chi$ given by (15). Features of the line element in this form are that 1) it is of the same form as the four dimensional case except for constant factors. Truncating the line element (23) by simply putting everything involving $\chi$ to zero, gives non vanishing $R_{\theta \theta}=R_{\varphi \varphi}=\alpha \beta$ and simple relationships between the product invariants, such as (17), are lost; however taking one of $\alpha$ or $\beta$ to vanish gives back (1). Going back to the five dimensional case $(23)_{\Theta=-r \exp (2 \varphi) / Y^{2}}$ gives $\Theta=-r \exp (2 \varphi) / Y^{2}$ so that there is essentially one independent object the scalar field $\varphi$, the projected Weyl tensor (20) now does not have a dependence on $\chi$ but does not seem to be simply expressible in terms of the scalar field $\chi$.2) $\chi$ does not appear explicitly in the metric, 3) THE scalar field takes exactly the same form as in the four dimensional case, 4)there are no non-vanishing Riemann or Ricci tensor $\chi$ indexed components, but there are Weyl tensor $\chi$ indexed components, also no component depends on $\chi$. The Ricci scalar is

$R=\left[(\alpha-\beta)^{2} r^{2}-4 \sigma r v(2 \sigma(1-\alpha \beta)+\alpha(\alpha-\beta))+4 \sigma^{2} v^{2}\left((1+2 \sigma)(1-\alpha \beta)+\alpha^{2}\right)\right] /\left(2 Y^{4}\right)$. 
Using the same projection vector (18), the acceleration and shear vanish, the extrinsic curvature take the same form as in (19) the expansion is

\section{And the shear is}

$$
\Theta=\frac{2 \alpha \sigma v-(\alpha+\beta) r}{Y^{2}}
$$

$$
\begin{aligned}
& \sigma(n)=-\frac{\sqrt{10}}{12} \Theta, \sigma_{a b}=-\frac{1}{6}\left(\delta_{a b}^{\theta \theta} g_{\theta \theta}+\delta_{a b}^{\varphi \varphi} g_{\varphi \varphi}\right) \Theta \\
& +\frac{1}{3}\left(\delta_{a b}^{r v} g_{r v}+\delta_{a b}^{r \chi} g_{r \chi}+\delta_{a b}^{v v} g_{v v}+\delta_{a b}^{\nu \chi} g_{v \chi}+\delta_{a b}^{\chi \chi} g_{\chi \chi} \frac{(\beta-\alpha) \gamma}{4 \sigma^{2}(1-\alpha \beta)-(\alpha-\beta) \gamma}\right) \Theta,
\end{aligned}
$$

The projected Weyl tensor (20) has ${ }_{\sigma=0}$ components and does not seem to be simply expressible. For $\sigma=0$ the coordinate transformation (22) and the metric (23) are not defined; defining $t \equiv v-r$, (14) reduces to

$$
d s^{2}=\frac{1}{1-\alpha \beta}\left(-d t^{2}+d r^{2}\right)+(r+\alpha \chi)(r+\beta \chi) d \Sigma_{2}^{2}+d \chi^{2}, \varphi=\frac{1}{2} \ln \left(\frac{r+\beta \chi}{r+\alpha \chi}\right),
$$

Which does not seem to further simplify. With respect to the vector field $X_{t} R i e^{2}=0$, many Lie derivatives vanish, in particular $X_{t} R i e^{2}=0$. For (27) when $\alpha=\beta$ the metric is flat.

\section{The second five dimensional solution}

In double null coordinates the second five dimensional generalization of the four dimensional solution is

$$
d s_{5}^{2}=-d u d v+Y^{2} d \Sigma_{2}^{2}+2 \beta(\chi)^{2} d \chi((1+2 \sigma) d v+d u)+\alpha(\chi)^{2} \gamma((1-2 \sigma) v-u)^{2} d \chi^{2},
$$

With $Y^{2}$ given by (4) and $\gamma$ is an arbitrary function which can be set to 1 or 0 . The line element is a scalar-Einstein solution when the ordinary differential equation $\beta \alpha_{\chi}=2 \alpha \beta_{\chi}$ is obeyed, which happens when either $\alpha$ and $\beta$ are independent constants or when $\beta(\chi)^{2}=A \alpha(\chi)$, A constant. In the $\beta=0, \gamma=1$ term the relative size of the $\mathrm{du}$ and $\mathrm{dv}$ contributions is fixed by the requirement $\beta=0, \gamma=1$. The scalar field is the same as for the four dimensional minimal scalar (2), the fifth component vanishing identically. The only case where there appears to be a homothetic Killing vector is when $\beta=0, \gamma=1$ and $\alpha$ is a constant when the situation is similar to the four dimensional case. In general this does not seem to be related to the first five dimensional generalizations (14), because of the factor of $1-\alpha \beta$ there. The Ricci scalar is

$$
R=\frac{32 \sigma^{2}\left((1+2 \sigma)^{2} \beta^{4} v^{2}+\left(\alpha^{2} \gamma^{2}+2(1+2 \sigma) \beta^{4}\right)+\beta^{4} u^{2}\right)}{((1+2 \sigma) v-u)^{2}((1-2 \sigma) v-u)^{2}\left(\alpha^{2} \gamma^{2}+4(1+2 \sigma) \beta^{4}\right)}
$$

The product invariants are again completely determined by the Ricci scalar, and are given by (17). The Gauss-Bonnet invariant and tensor vanish in $\mathbf{U}$. In d greater than 4 they are objects constructed from products of the Riemann tensor. Here the Gauss-Bonnet invariant (43) and tensor (44) vanish. Projecting using (18) the acceleration and rotation vanish and the expansion, extrinsic curvature and shear are

$$
\Theta=\frac{8 \sigma \beta^{2}}{(u-(1-2 \sigma) v)\left(\alpha^{2} \gamma^{2}+4(1+2 \sigma) \beta^{4}\right)}, K_{\theta \theta}=\sin (\theta)^{-2} K_{\varphi \varphi}=\frac{u-(1+2 \sigma) v}{8(u-(1-2 \sigma) v)} \Theta,
$$

$\sigma_{a b}=-\frac{1}{3}\left(\frac{\alpha^{4} \gamma^{4}+4(1+2 \sigma) \alpha^{2} \gamma^{2} \beta^{4}-1}{\alpha^{2} \gamma^{2}+4(1+2 \sigma) \beta^{4}} \delta_{a b}^{\chi \chi}+2 \delta_{a b}^{(u v)} g_{u v}+\delta_{a b}^{u \chi} g_{u \chi}+\delta_{a b}^{v \chi} g_{v \chi}-\frac{1}{8}\left(\delta_{a b}^{\theta \theta} g_{\theta \theta}+\delta_{a b}^{\varphi \varphi} g_{\varphi \varphi}\right)\right) \Theta$

All of which vanish for $\beta=0$. The shear scalar and the projected Weyl tensor are independent of $\chi$ and do not seem to simply factor.

\section{The third five dimensional solutions}

The solution is

$$
d s_{5}^{2}=\sqrt{\chi}\left\{-d u d v+Y^{2} d \Sigma_{2}^{2}\right\}+d \chi^{2}, \varphi_{2}=\frac{1}{2} \sqrt{\frac{3}{2}} \ln \chi,
$$

With the term in the brackets given by (1) and $\varphi_{1}$ given by (5). The con- formal factor $\sqrt{\chi}$ fixed by requirement $R_{\theta \theta}=0$. This is a solution for two scalar fields to the field equations

$$
R_{a b}=2 \varphi_{1, a} \varphi_{1, b}+2 \varphi_{2, a} \varphi_{2, b} .
$$

The solution has a conformal Killing vector with conformal factor and expansion

$$
V^{a}=c\left[-2 u, 0,0,-2 v,-\frac{8}{3} \chi+A \chi^{\frac{1}{4}}\right], C f=\frac{1}{5} \Theta=-\frac{c}{12}\left(32-3 A \chi^{-\frac{3}{4}}\right),
$$

The norm and acceleration of this vector are not simple, the shear and the fifth dimensional components $\{u, \chi\}$ and $\{v, \chi\}$ of the vorticity are non- vanishing. The Nother current is related to the symmetry properties of a Lagrangian and is typically a vector. Here the Nother current is

$$
j_{a} \equiv i\left(\varphi^{*} \partial_{a} \varphi-\varphi \partial_{a} \varphi^{*}\right)=\varphi_{2} \varphi_{1 a}-\varphi_{1} \varphi_{2 a} .
$$

Here the Nother current is

$$
j_{a}=\frac{\sqrt{6}}{8}\left[\frac{\sigma \sqrt{\chi} \ln (\chi)}{Y^{2}}\left(-v \delta_{a}^{u}+u \delta_{a}^{v}\right)-\frac{1}{\chi} \ln \left(\frac{(1-2 \sigma) v-u}{(1+2 \sigma) v-u}\right)\right]
$$

The size of Nother current is

$$
j_{a}^{2}=-\frac{1}{2 \sqrt{\chi}} R^{(4)} \varphi_{2}^{2}+\frac{3}{8 \chi^{2}} \varphi_{1}^{2},
$$

And this is of undetermined sign, so that the Nother current can be time like, null, or space like.

The scalar invariants are

$$
\begin{aligned}
& R=\frac{R^{(4)}}{\sqrt{\chi}}+\frac{3}{4 \chi^{2}}, \text { Weyl }^{2}=\frac{11 R^{(4)^{2}}}{6 \chi}, \\
& \operatorname{Rie}^{2}=\frac{3 R^{(4)^{2}}}{\chi}-\frac{R^{(4)}}{4} \chi^{-\frac{5}{2}}+\frac{21}{32 \chi^{4}}, \text { Ricci }^{2}=\frac{R^{(4)^{2}}}{\chi}+\frac{9}{16 \chi^{4}} .
\end{aligned}
$$

Where $R^{(4)}$ is given by (7). Using the projection vector (18), the acceleration and rotation vanish and the expansion, extrinsic curvature, shear, and projected Weyl tensor, which for this metric is the same as the electric part of the Weyl tensor are

$$
\begin{gathered}
\Theta=\frac{1}{\chi}, \sigma(n)=\frac{1}{6 \chi} \\
K_{a b}=\frac{1}{4}\left(\delta_{a b}^{u v} g_{u v}+\delta_{a b}^{\theta \theta} g_{\theta \theta}+\delta_{a b}^{\varphi \varphi} g_{\varphi \varphi}\right), \sigma_{a b}=-\frac{1}{3} K_{a b} \\
E_{a b}=\frac{\sigma^{2} \chi}{6 Y^{4}}\left(-v^{2} \delta_{a b}^{u u}+u v \delta_{a b}^{(u v)}-u^{2} \delta_{a b}^{v v}\right)+\frac{\sigma^{2} u v \sqrt{\chi}}{6}\left(\delta_{a b}^{\theta \theta} g_{\theta \theta}+\delta_{a b}^{\varphi \varphi} g_{\varphi \varphi}\right)
\end{gathered}
$$

\section{Five dimensional vaidya spacetime}

A generalization of Vaidya's spacetime to five dimensions is

$$
d s^{2}=\sqrt{\chi}\left\{-\left(1-\frac{2 m(v, \chi)}{r}\right) d v^{2}+2 d v d r+r^{2} d \Sigma_{2}^{2}\right\}+d \chi^{2},
$$

Choosing the requirement that $R_{\theta \theta}=0$ fixes the conformal factor as $\sqrt{\chi},(39)$ has Ricci tensor

$$
R_{v v}=\frac{2 m_{v}}{r^{2}}-\frac{\left(\chi m_{\chi}\right)_{\chi}}{r \sqrt{\chi}}, R_{r v}=\frac{m_{\chi}}{r^{2}}, R_{\chi \chi}=\frac{3}{4 \chi^{2}},
$$

The $m$ dependence of $m$ means that the four dimensional stress 
is no longer that of a null radiation field. The $R_{\gamma \gamma}$ component is non vanishing even when $m$ is independent of $\mathrm{m}$. The Ricci scalar and product invariants are

$$
R=\frac{3}{4 \chi^{2}}, \text { Weyl }^{2}=\frac{48 m^{2}}{r \chi}, \text { Ricci }^{2}=R^{2}, \text { Rie }^{2}=\text { Weyl }^{2}+\frac{7}{6} R^{2}
$$

These do not explicitly involve derivatives of $m$ with respect to either $v$ or $\chi$. Using the projection vector (18), the acceleration and rotation vanish and the expansion, extrinsic curvature, shear, and projected Weyl tensor, which for this metric is the same as the electric part of the Weyl tensor are

$$
\begin{gathered}
\Theta=\frac{1}{\chi}, K_{v r}=\frac{1}{4 \sqrt{\chi}}, K_{\theta \theta}=\frac{r^{2}}{4 \sqrt{\chi}}, \\
\sigma=\frac{1}{6 \chi}, \sigma_{r v}=-\frac{1}{12 \sqrt{\chi}}, \sigma_{\theta \theta}=-\frac{r^{2}}{12 \sqrt{\chi}}, \sigma_{v v}=\frac{1}{12 r \sqrt{\chi}}\left(12 \chi m_{\chi}+r-2 m\right), \\
E_{v V}=-\frac{2 \chi^{1} 4}{3 r}\left(\chi^{\frac{1}{4}} m_{\chi}\right)_{\chi}-\frac{2}{3 r^{2}} m_{v} .
\end{gathered}
$$

\section{The gauss-bonnet invariant}

The Gauss-Bonnet invariant

$$
G B=R_{c d e f} R^{c d e f}-4 R_{c d} R^{c d}+R^{2}
$$

And Gauss-Bonnet tensor

$$
G B_{a b}=4 R_{a c d e} R_{b}^{c d e}-8 R_{c d} R_{a b}^{c d}-8 R_{a c} R_{b}^{c}+4 R R_{a b}-g_{a b} G B
$$

Vanish for the first two solutions (14), (28), and non-vanishing and long for third scalar solution (31) and the Vaidya solution (39). There does not seem a way of reinterpreting the scalar-Einstein solutions as Einstein-Gauss- Bonnet solutions. For the Einstein-Gauss-Bonnet field equations

$$
G_{a b}+k G B_{a b}=0,
$$

There is the simple solution

$$
d s_{5}^{2}=\exp \left( \pm \frac{\chi}{\sqrt{k}}\right) d s_{4 \text { flat }}^{2}+d \chi^{2},
$$

This is the same as the Randall-Sundrum (1999) metric ${ }^{11}$ with $2 \sqrt{k}= \pm l$, and this allows reinterpretation of the coupling between the Einstein tensor and the Gauss-Bonnet tensor as the cosmological constant. There is the modified Schwarzschild spacetime

$$
d s_{5}^{2}=\exp \left(\frac{\chi}{\sqrt{\alpha}}\right) d s_{4 \text { Schwarzschild }}^{2}+d \chi^{2} .
$$

This has invariants:

$$
R=-\frac{5}{\alpha}, \text { Ricci }^{2}=\frac{1}{5} R^{2}, \text { Weyl }^{2}=\frac{48 m^{2} \exp \left(-\frac{2 \chi}{\sqrt{\alpha}}\right)}{r^{6}}, \text { Rie }^{2}=\frac{1}{2} \text { Ricci }^{2}+\text { Weyl }^{2}
$$

This is a solution to any of three field equations:

the vacuum Bach equations $P_{a b}:=2 R_{a ; a b}-2 R R_{a b}+g_{a b}\left(R^{2} / 2-2 \square R\right), G_{a b}+p P_{a b}=0$
The Einstein-Pauli equations

$P_{a b}:=2 R_{: a ; b}-2 R R_{a b}+g_{a b}\left(R^{2} / 2-2 \square R\right), G_{a b}+p P_{a b}=0$ with $\left.p=-3 \alpha / 5,3\right)$ the Einstein-Gauss-Bonnet equations (45) with $\alpha=k$ and stress $T a_{a b}=-\delta_{a b}^{\chi \chi}$ Weyl $^{2}$.

\section{Conclusion}

There at least three criteria one could use to test interaction with the fifth dimension. The first is explicit interaction. The Einstein tensor for a minimal scalar field is $G_{a b}=2 \varphi_{a} \varphi_{b}-\varphi_{c}^{2} g_{a b}$, so that with respect to a vector field $v_{a}$ there is the momentum transfer $\pi_{a}=v^{b} G_{a b}=2 \varphi_{a} \varphi v-v_{a} \varphi_{c}^{2}$. Such a momentum transfer seems unavoidable for non vanishing scalar field because of the metric (or second) term in the Einstein tensor; whether this is good or bad depends on ones point of view. It is good if one simply wants any indication of transfer of information. It is bad if one wants only gravity, in the sense that $R_{5 a}=0$, to be present in the fifth dimension, because there will also be the scalar field present. For the above examples it is also bad because there is no neat way of characterizing the energy transfer, it is not even clear when it will be time like, null, or space like. The second is implicit interaction. By this is meant that the four dimensional metric takes a different form than would be expected from four dimensional theories and that this difference can somehow be measured. To illustrate this consider (23), except for the factor of $1-\alpha \beta$ the metric truncated to four dimensions would be of the same form as (1). Roughly the $d v^{2}$ term suggests a change in the null velocity from $c \rightarrow c / \gamma(1-\alpha \beta)$; how- ever other metric terms change as well and it turns out not to be possible to have only the null velocity change occurring. For $\sigma=0$, given by equation (27), similar problems apply. The third is Nother criteria. One could image scalar fields as in some way corresponding to a quantum mechanical wave function of some part of a system. A quantum mechanical interaction might be indicated by a non vanishing Nother current between one part of the system and another. To model this one would need exact solutions for two or more scalar fields. It turns out to be simple to produce solutions for linear combinations of scalar fields, however these have vanishing Nother current. Spherical symmetry imposes a high degree of symmetry making finding solutions with a non vanishing Nother current hard to find. An example with a non vanishing Nother current is (31); but prop- erties of this solution include 1) the two scalar fields are disconnected, in the sense that one scalar field de- pends on one set of coordinates $\{r, v\}$ and the other depends on $\{\chi\}, 2$ ) the current can be time like, null or spacelike, and 3) there is no simple way of characterizing what happens to any Nother charge. Another problem in general with the Nother criteria is that in quantum cosmology the high degree of symmetry means that there are no Nother currents. To conclude the five dimensional scalar-Einstein equations provide simple exact solutions with which to discuss interaction with the fifth dimension, how ever their interpretation is difficult.

\section{Acknowledgements}

None.

\section{Conflicts of interest}

Author declares there is no conflict of inertest.

\section{References}

1. Fischer IZ. Scalar mesostatic field with regard for gravitational effects translated Zloshchastiev. Zhurnal Experimental'noj $i$ Teoreticheskoj Fiziki. 1947;18:636-640. 
2. Roberts MD. Spacetime Exterior to a Star. Conell University Library, USA, 1998. p. 1-14.

3. Chase JE. Event Horizons in static scalar-vacuum space-times. Communications in Mathematical Physics. 1970;19(4):276-288.

4. Roberts MD. Spherically Symmetric Fields in Gravitational Theory. Ph.D.Thesis, University of London, England. 1986.

5. Roberts MD. Scalar Field Counter-Examples to the Cosmic Cen- sorship Hypothesis. General Relativity and Gravitation. 1989;21(9):907-939.

6. Roberts MD. Imploding Scalar Fields. Journal of Mathematical Physics. 1996;37(9):4557-4573.

7. Choptuik MW. Universality and Scaling in Gravitational Collapse of a Massless Scalar Field. Physical Review Letters. 1993;70(9):9-12.

8. Roberts MD. Quantum Imploding Scalar Fields. Conell University Library, USA; 2017. p. 1-12.

9. Pavsic M, Tapia V. Resource Letter on the Geomtric Results for
Embeddings and Branes. Conell University Library, USA; 2001. p. $1-40$.

10. Wuensch D. The Fifth Dimension: Theodor Kaluza's ground breaking idea. Annalen der Physik. 2003;12(9):519-542.

11. Randall L, Sundrum R. Large Mass Hierarchy from a Small Extra Dimension. Physical Review Letters. 1999;83(17).

12. Randall L, Sundrum R. An Alternative to Compactification. Physical Review Letters. 1999;83(23).

13. Wesson PS, Mashhoon B, Liu H. The (Im) possibility of detecting a fifth dimension. Modern Physics Letters A. 19977;12(30):2309-2316.

14. Roberts MD. New Five Dimensional Spherical Vacuum Solutions. Conell University Library, USA; 2009. p. 1-16.

15. Carminati J, McLenaghan RG. Algebraic invariants of the Riemann tensor in a four-dimensional Lorentzian space. Journal of Mathematical Physics. 1991;32(11):3135-3140. 